\title{
Apoptotic spermatogenic cells can be energy sources for Sertoli cells
}

\author{
Weipeng Xiong, Haikun Wang, Hui Wu, Yongmei Chen and Daishu Han \\ Department of Cell Biology, School of Basic Medicine, Peking Union Medical College, Institute of Chinese Basic \\ Medical Sciences, Chinese Academy of Medical Sciences, 5 Dong Dan San Tiao, Beijing 100005, People's Republic \\ of China
}

Correspondence should be addressed to D Han; Email: dshan@ibms.pumc.edu.cn

\begin{abstract}
Apoptotic spermatogenic cells and residual bodies are phagocytosed and degraded by Sertoli cells during mammalian spermatogenesis. The meaning of this event remains to be clarified. In this report, we demonstrate that apoptotic spermatogenic cells and residual bodies can be used to produce ATP by Sertoli cells after phagocytosis of them. Sertoli cells produced the highest level of ATP compared with other testicular cells. Phagocytosis assay in vitro showed that engulfment of apoptotic spermatogenic cells increases ATP production by Sertoli cells. The increased ATP production was detected in seminiferous tubules at the stages where phagocytosis occurs. Induced apoptosis of spermatogenic cells in vivo increased ATP production in seminiferous tubules. The augmentation of ATP production both in vitro and in vivo associated with the lipid formation in Sertoli cells after phagocytosis of apoptotic spermatogenic cells. The lipid $\beta$-oxidation was a predominant pathway to produce ATP in Sertoli cells. We conclude that after phagocytosis by Sertoli cells, apoptotic spermatogenic cells are degraded to form lipids that are then used to produce ATP. The results suggest that apoptotic spermatogenic cells can be energy sources for Sertoli cells that may define a novel meaning of spermatogenic cell death.
\end{abstract}

Reproduction (2009) 137 469-479

\section{Introduction}

Mammalian spermatogenesis is a complex process comprising a series of events, including proliferation and differentiation of spermatogonia, meiotic division of spermatocytes and morphogenic maturation of spermatids. In this process, more than $75 \%$ of developing spermatogenic cells are estimated to undergo apoptosis before they mature into spermatozoa under physiological condition (Oakberg 1956, Huckins 1978, Johnson et al. 1983, Dym 1994). In the last stage of spermatogenesis, the cytoplasmic portions of elongated spermatids are shed and form residual bodies before extrusion of differentiated sperm into the lumen of the seminiferous tubules (Kerr \& de Kretser 1974). Sertoli cells endocytose and degrade the residual bodies and apoptotic spermatogenic cells, which is necessary for healthy spermatogenic cells to proceed through spermatogenesis (Chemes 1986, Pineau et al. 1991, Miething 1992). Apoptotic spermatogenic cells are phagocytosed by Sertoli cells in a phosphatidylserine (PS)-mediated manner, in which class B of scavenger receptor type I (SR-BI) in Sertoli cells functions as a PS receptor (Shiratsuchi et al. 1997, 1999, Kawasaki et al. 2002, Nakagawa et al. 2005). We recently demonstrated that members of the Tyro3 subfamily of receptor tyrosine kinases (TYRO3, AXL,
MER) play roles in regulating phagocytosis of apoptotic spermatogenic cells by Sertoli cells (Xiong et al. 2008). It is unclear why such a large proportion of differentiating spermatogenic cells undergoes apoptosis and is discarded. A reasonable explanation is that the number of spermatogenic cells that Sertoli cells can support for maturation is limited, and the cells beyond this number should be removed. Three explanations for how the removal of apoptotic spermatogenic cells contributes to spermatogenesis have been proposed (Nakanishi \& Shiratsuchi 2004): 1) elimination of apoptotic germ cells provides appropriate spaces in the seminiferous epithelium for normal spermatogenesis, 2) apoptotic cells need to be removed before their noxious contents leak out and poison healthy cells, and 3) engulfment of apoptotic spermatogenic cells enables Sertoli cells to produce factors necessary for spermatogenesis. The relevance of the phagocytic clearance of dead germ cells to the functions of Sertoli cells has not been intensely analyzed. Our present study focuses on the meaning of the phagocytosis of apoptotic spermatogenic cells by Sertoli cells for spermatogenesis.

In testes, the lipid droplets within Sertoli cells were described as early as the cells were discovered. However, the formation and fate of the lipid droplets have not been extensively studied. The temporal 
relationship between the phagocytosis of residual bodies and the increased lipid droplets in Sertoli cells suggests that the lipid droplets could result from the breakdown products of phagocytosed residual bodies (Kerr et al. 1984, Ueno \& Mori 1990, Sasso-Cerri et al. 2001). We recently demonstrated in vitro that Sertoli cells can use engulfed apoptotic spermatogenic cells to form the lipid droplets (Wang et al. 2006). In mice, spermatogenesis can be compromised by inactivation of genes involved in lipid metabolism, suggesting that appropriate lipid metabolism is critical for male reproduction (Chung et al. 2001). The lipids play numerous biological functions in mammals, including as energy substrates to produce ATP. Although both lipids and glucoses can be energy sources, glycolysis is an active ATPproducing pathway in the majority of cell types, whereas the lipids function mainly in the storage of energy. Only minority cell types, such as adipocytes and myocardial cells, actively utilize fatty acids as major energy substrates under physiological conditions (Saddik \& Lopaschuk 1991, Klaus 2004). Our present study demonstrates that Sertoli cells, the only somatic cells within the seminiferous epithelium, predominantly utilize lipids to produce energy through the $\beta$-oxidation pathway. Interestingly, the lipids result from the degradation of ingested apoptotic spermatogenic cells and residual bodies. Our finding indicates that phagocytic clearance of apoptotic spermatogenic cells provides energy substrates to Sertoli cells.

\section{Results}

\section{Sertoli cells produce the highest ATP level in testis}

During spermatogenesis, Sertoli cells provide physical supports for germ cells to move to the adluminal compartment from the basal compartment, and for elongated spermatids to be released into the lumen of seminiferous tubules. These functions of Sertoli cells consume more energy. Therefore, we expected that Sertoli cells should produce high levels of ATP. To examine this speculation, we measured ATP levels in different types of testicular cell (interstitial cells, Sertoli cells and spermatogenic cells), and found that Sertoli cells produce a significantly higher level of ATP than interstitial cells and spermatogenic cells. As shown in Fig. 1, although a comparable ATP level was observed in spermatogenic cells and interstitial cells, a fivefold higher level of ATP in Sertoli cells was detected compared with the spermatogenic cells or interstitial cells. Considering Sertoli cells are epithelial cells and have phagocytic ability in testis, we asked whether the high ATP level is a common phenomenon in epithelial cells and phagocytes. Therefore, we compared ATP production by professional phagocytes, macrophages and epithelial cells, epididymal cells. The result showed that the ATP level in Sertoli cells is about
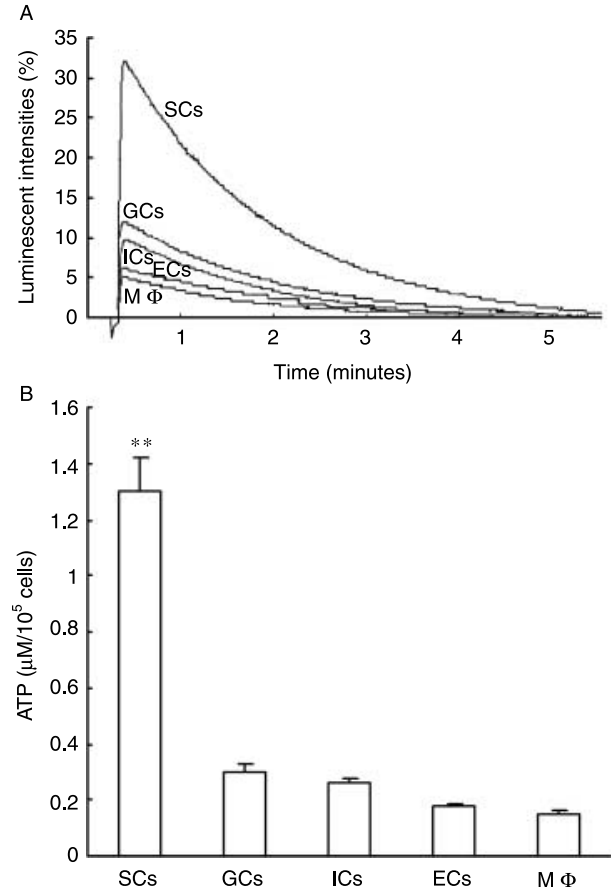

Figure 1 Comparison of ATP levels in testicular cells. (A) Representative profiles of cellular ATP bioluminescent intensities in primary Sertoli cells (SCs), germ cells (GCs), interstitial cells (ICs), epididymal cells

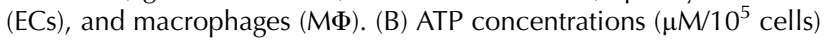
were calculated based on the bioluminescent intensities. A significant high ATP level was only observed in Sertoli cells. The data are represented as mean \pm S.E.M. of five experiments. ${ }^{* *} P<0.01$.

sixfold higher compared with those in macrophages and epididymal cells. These results indicate that the high ATP level is specifically produced by Sertoli cells.

\section{Phagocytosis of apoptotic spermatogenic cells increases ATP production by Sertoli cells}

Lipids are common energy substrates for the production of ATP within cells. Most of differentiating spermatogenic cells are estimated to undergo apoptosis under physiological conditions and then phagocytic clearance by Sertoli cells (Nakanishi \& Shiratsuchi 2004), which results in the formation of lipids in Sertoli cells (Chemes 1986, Wang et al. 2006). We speculated that apoptotic spermatogenic cells might be an energy source for Sertoli cells. To examine this possibility, we analyzed the correlation of the ATP production and the phagocytosis of apoptotic spermatogenic cells by Sertoli cells. As expected, we found that Sertoli cells produce a significantly higher level of ATP after phagocytosis of apoptotic germ cells. At $24 \mathrm{~h}$ after co-culture with apoptotic cells, the ATP level in Sertoli cells was 3.3fold higher than that in single cultured Sertoli cells (Fig. 2A). In a parallel assay, macrophages did not increase ATP production after the phagocytosis of apoptotic spermatogenic cells. As controls, interstitial 

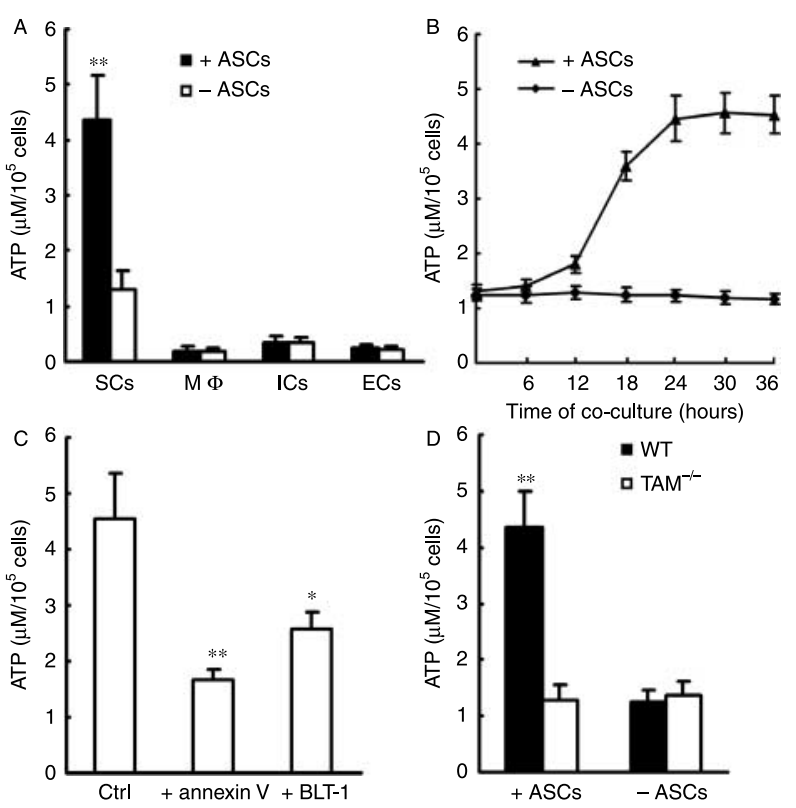

Figure 2 Increased ATP level in Sertoli cells after phagocytosis of apoptotic spermatogenic cells. (A) ATP levels in Sertoli cells (SCs),

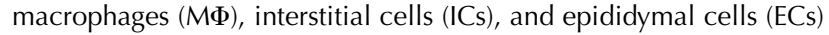
cultured with and without apoptotic spermatogenic cells (ASCs). A significantly increased ATP level was observed in Sertoli cells after co-culture with ASCs. (B) Dynamics of ATP production by Sertoli cells during the co-culture with ASCs. (C) Inhibition of ATP production in Sertoli cells co-cultured with ASCs by annexin V and BLT-1. The two inhibitors of phagocytosis significantly inhibit ATP production by Sertoli cells. (D) Comparison of ATP levels in wild-type (WT) and $\mathrm{TYRO}^{-1-} \mathrm{AXL}^{-1-} \mathrm{MER}^{-1-}\left(\mathrm{TAM}^{-1-}\right)$ Sertoli cells. TAM ${ }^{-1-}$ Sertoli cells loss the phagocytic ability in phagocytosis of apoptotic spermatogenic cells. The data are represented as mean \pm s.D. of three experiments. ${ }^{*} P<0.05 ;{ }^{* *} P<0.01$.

Leydig cells and epididymal epithelial cells produced only low ATP levels comparable before and after co-culture with apoptotic spermatogenic cells. The results indicate that Sertoli cells produce the higher level of ATP by engulfing apoptotic germ cells. Analysis on the kinetics of ATP production by Sertoli cells during the co-culture with apoptotic spermatogenic cells showed a marked increase in the ATP production at $18 \mathrm{~h}$, and the ATP level reached a plateau at $24 \mathrm{~h}$ and thereafter (Fig. 2B).

To further confirm that phagocytosis of apoptotic spermatogenic cells increases ATP production in Sertoli cells, we measured the ATP level of Sertoli cells in the presence of phagocytosis inhibitors in assay. Previous studies showed that a PS binding protein (annexin V) and a inhibitor of SR-BI (block lipid transporter-1 (BLT-1)) inhibit the phagocytosis of apoptotic spermatogenic cells by Sertoli cells (Maeda et al. 2002, Nakanishi \& Shiratsuchi 2004, Nakagawa et al. 2005). In the presence of annexin $\mathrm{V}$ or/and BLT-1 during the co-culture with apoptotic germ cells, the ATP production by Sertoli cells was markedly inhibited (Fig. 2C). Our recent study demonstrated that phagocytic activity of Sertoli cells
Table 1 ATP production by Sertoli cells after phagocytosis of different targets.

\begin{tabular}{lc}
\hline Phagocytic targets & ATP levels $\left(\mu \mathrm{M} / 10^{5} \text { cells }\right)^{\mathrm{a}}$ \\
\hline Apoptotic spermatogenic cells & $4.56 \pm 0.33^{*}$ \\
Living spermatogenic cells & $1.41 \pm 0.12$ \\
Bacteria $(E$. coli TOP 10) & $1.36 \pm 0.07$ \\
Latex beads & $1.42 \pm 0.08$ \\
No (control) & $1.43 \pm 0.11$ \\
\hline
\end{tabular}

Score with asterisks is significantly different from that of control. $* P<0.01$.

${ }^{\mathrm{a}}$ At $24 \mathrm{~h}$ after co-culture with phagocytic targets, ATP levels in Sertoli cells were measured. Each assay was repeated five times and the values are presented as mean \pm S.D.

was abolished by lacking the Tyro3 family of receptor tyrosine kinases (TYRO3, AXL, MER; Xiong et al. 2008). Here, we examined ATP level in the mutant Sertoli cells. As expected, they produced significantly low ATP compared with wild-type Sertoli cells after the phagocytosis of apoptotic germ cells (Fig. 2D). At $24 \mathrm{~h}$ after co-culture with apoptotic spermatogenic cells, the level of ATP in wild-type Sertoli cells was 3.5-fold higher than that in $\mathrm{TYRO}^{-1-} \mathrm{AXL}^{-1-} \mathrm{MER}^{-1-}\left(\mathrm{TAM}^{-1-}\right)$ Sertoli cells. In contrast, single-cultured wild-type and mutant Sertoli cells did not show a difference in ATP production.

To determine whether the increased ATP production by Sertoli cells was specifically due to the phagocytosis of apoptotic germ cells, we compared the ATP production in Sertoli cells after co-culturing with other phagocytic targets: living spermatogenic cells, inactivated bacteria (Escherichia coli TOP 10) and latex beads. The increased ATP level was only observed in Sertoli cells after co-culture with apoptotic spermatogenic cells, and no obvious change in ATP production was detected in Sertoli cells before and after co-culture with other phagocytic targets (Table 1 ). The results indicate that only the co-culture with apoptotic spermatogenic cells increased ATP production by Sertoli cells.

\section{ATP production correlates with the lipid formation in Sertoli cells}

To determine whether the lipids resulting from engulfment of apoptotic spermatogenic cells are energy substrates in Sertoli cells, we examined the correlation between the lipid formation and ATP production during co-culture of Sertoli cells with apoptotic germ cells. The lipid droplets and ATP levels were determined at 0, 12, 24,48 , and $72 \mathrm{~h}$ during the co-culture. The lipid droplets were visualized by Oil Red O (ORO) staining. Only a few small lipid droplets were observed in single-cultured Sertoli cells (Fig. 3A); however, many large lipid droplets were formed in Sertoli cells at $24 \mathrm{~h}$ after co-culture with apoptotic germ cells (Fig. 3B). The lipid droplets were quantified by the area ratio of the lipid droplets to Sertoli cell nucleus. Compared to control, the ratio increased dramatically at $12 \mathrm{~h}$, and reached a plateau at $48 \mathrm{~h}$ after 
the co-culture (Fig. 3C). By contrast, the lipid droplets in single-cultured Sertoli cells remained at a low and consistent level during the culture. The kinetics of ATP production by Sertoli cells well matched the lipid droplet formation (Fig. 3D). The ATP level in Sertoli cells increased dramatically at $12 \mathrm{~h}$, and reached a plateau at $24 \mathrm{~h}$ after the co-culture. By contrast, ATP in singlecultured Sertoli cells remained at a low level.

Furthermore, after removing apoptotic spermatogenic cells from the co-culture, the amount of lipid droplets in Sertoli cells decreased rapidly (Fig. 3E). The area ratio of the lipid droplets to Sertoli cell nucleus dropped back to a baseline level at $48 \mathrm{~h}$ after removal of the germ cells and did not further decrease thereafter. Consistent with the reduction in the lipid droplets, ATP production in Sertoli cells decreased gradually (Fig. 3F). By contrast, both the lipids and ATP production remained at high levels in Sertoli cells without removal of the germ cells. The results indicate that the ATP production in Sertoli cells correlates closely to the lipid droplet formation during the co-culture between Sertoli cells and apoptotic spermatogenic cells, suggesting that the lipids might be used as energy substrates by Sertoli cells.

\section{Phagocytosis of residual bodies and apoptotic spermatogenic cells by Sertoli cells in vivo increases ATP production in seminiferous tubules}

The most cytoplasmic portions of elongated spermatids are shed to form residual bodies at stage IX, which are then engulfed by Sertoli cells. Previous studies demonstrated that the lipid droplets accumulate within Sertoli cells at stages IX-XII where the phagocytosis of residual bodies take places (Kerr \& De Kretser 1975, Ueno \& Mori 1990). To examine the correlation of lipid formation and ATP production in seminiferous tubules, we separated four fragments of the staged tubules (Fig. 4A). As shown in Fig. 4B, the highest level of ATP was detected in the fragments of tubules at stages IX-XI and XII-I, then the ATP level slightly decreased at stages II-VI, and the lowest level of ATP was observed at stages VII-VIII. The results suggest that the phagocytosis of residual bodies by Sertoli cells results in the augmentation of ATP production in seminiferous tubules.

To further analyze in vivo the correlation among apoptosis of spermatogenic cells, lipid formation and ATP production in seminiferous tubules, we induced

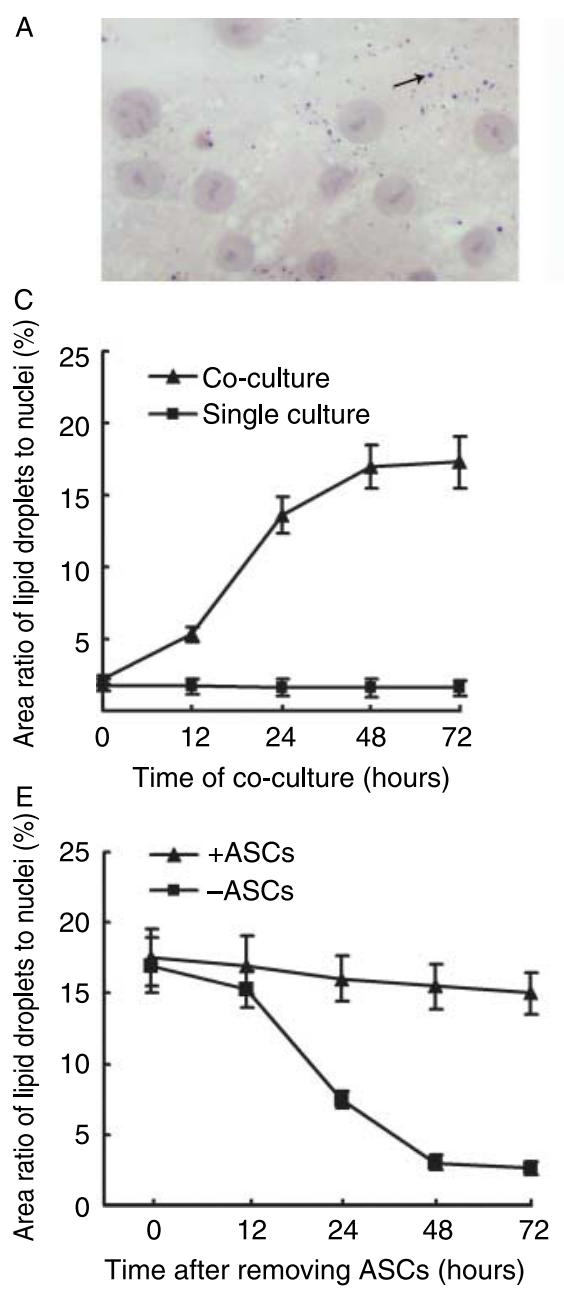

B

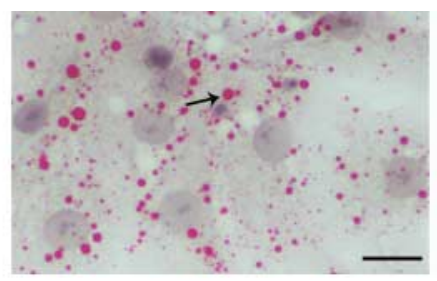

D

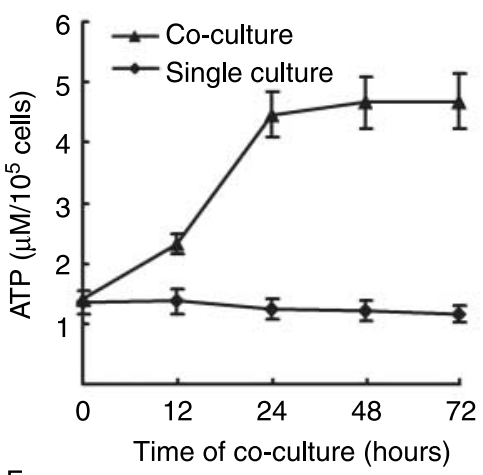

$\mathrm{F}$

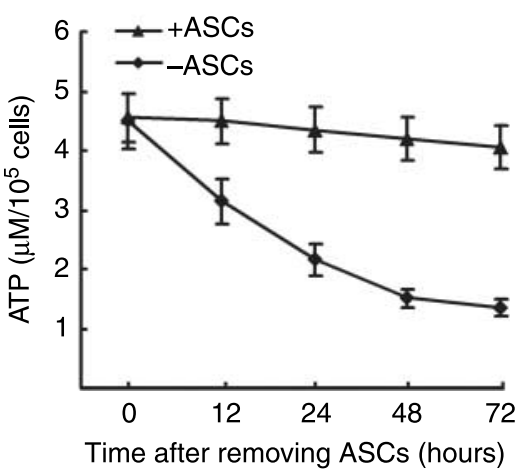

Figure 3 Correlation between the lipid formation and ATP production in Sertoli cells. (A), (B) Representative images of lipid droplets (arrows) in Sertoli cells before and after phagocytosis of apoptotic spermatogenic cells (ASCs). Abundant lipid droplets were observed in Sertoli cells at $24 \mathrm{~h}$ after co-culture with ASCs (right panel). Bar $=20 \mu \mathrm{m}$. (C) Dynamics of the lipid droplet formation in Sertoli cells during co-culture with ASCs. (D) Dynamics of ATP production by Sertoli cells during co-culture with ASCs. (E) The lipid droplet formation in Sertoli cells after removing ASCs at $48 \mathrm{~h}$ after co-culture. (F) Dynamics of ATP level in Sertoli cells after removing ASCs from the co-cultures. Each datum was expressed as mean \pm s.D. of three experiments. 
apoptosis of spermatogenic cells in vivo by an anticancer drug, busulfan (Sigma). After administration of busulfan in mice, the number of TUNEL-positive spermatogenic cells in seminiferous tubules dramatically increased at week 1 compared with that of control (week 0), and reached a plateau at week 3 , then sharply decreased at week 5 when most of the seminiferous tubules are depleted of germ cells (Fig. 4C). The number of lipid droplets in Sertoli cells increased significantly after the injection of busulfan (Sigma; Fig. 4D). A peak number of lipid droplets was observed at 3-4 weeks and decreased at week 5. Consistent with the kinetics of germ cell apoptosis and lipid droplet formation in Sertoli cells, the ATP level of seminiferous tubules was increased markedly at 2 weeks after the injection and reached a peak level at week 3, then decreased at week 5 (Fig. 4E). These results suggest that the induced apoptotic spermatogenic cells are phagocytosed by Sertoli cells and used to produce ATP in vivo.

\section{Lipid $\beta$-oxidation is a major pathway to produce ATP in Sertoli cells}

Lipid $\beta$-oxidation and glycolysis are two universal metabolic pathways for the catabolism of lipids and glucoses to produce energy within cells. Each cell type predominantly utilizes one of the two energy substrates for ATP production. To assess which metabolic pathway is used to produce energy in Sertoli cells, we examined the expression pattern of key enzyme genes involved in $\beta$-oxidation and glycolysis in Sertoli cells during the phagocytosis of apoptotic spermatogenic cells. Quantitative RT-PCR analysis showed that the expression of long-chain acyl-CoA dehydrogenase (LACD) was increased four times in Sertoli cells after phagocytosis of apoptotic spermatogenic cells (Fig. 5A). However, the gene expression of the other enzymes participating in the glycolysis and $\beta$-oxidation was not changed significantly (Fig. 5B). To further confirm the major metabolic pathway to produce ATP in Sertoli cells, the lipid $\beta$-oxidation and glycolysis were blocked by a LACD inhibitor (2-mercaptoacetate (2-MP); Sinopharm Co., Ltd, Beijing, China) and a hexokinase inhibitor (2-deoxyglucose (2-DG), Sigma) respectively. In the presence of 2-MP $(0.5 \mathrm{mM})$, ATP level in Sertoli cells was decreased by fourfold at $24 \mathrm{~h}$ after co-culture with apoptotic spermatogenic cells compared with the control (Fig. 5C). By contrast, as glycolysis pathway was inhibited by 2-DG $(10 \mathrm{mM})$ in the phagocytosis assay, the ATP level in Sertoli cells did not decrease compared with the control. These results suggest that Sertoli cells utilize predominantly the long-chain fatty acids (LCFAs) to produce ATP. Given that the lipids formed in Sertoli cells after engulfment of apoptotic spermatogenic cells are mainly consisted of LCFAs, we conclude that apoptotic germ cells are an energy source of Sertoli cells.

\section{Differentiated functions of Sertoli cells cultured in vitro}

To determine whether primary cultured Sertoli cells can retain their differentiated functions, we examined the phagocytotic ability of Sertoli cells through ingesting latex beads, and expression of two functional markers of Sertoli cells, androgen binding protein (ABP) and androgen receptor (AR), during in vitro culture. As shown in Fig. 6A, there was no difference in the percentage of Sertoli cells that ingested latex beads at 7 and 14 days after culture, which were 25.6 and $26.1 \%$ respectively. By contrast, treatment with cytochalasin B resulted in a marked decrease in the phagocytosis of latex beads by the Sertoli cells. Moreover, mRNA levels of $A b p$ and $A r$ in the cultured Sertoli cells were detected by real-time RT-PCR (Fig. 6B). These data suggest that the Sertoli cells retain their major differentiated functions during culture.

\section{Discussion}

Phagocytic clearance of apoptotic spermatogenic cells and residual bodies by Sertoli cells is necessary for efficient production of sperm (Maeda et al. 2002). In this study, we demonstrate that phagocytosis of apoptotic spermatogenic cells results in the formation of lipids, which are further metabolized to produce ATP in Sertoli cells. This energy source could be physiologically important for Sertoli cells to support spermatogenesis.

Sertoli cells provide essential physical and tropic support for the development of spermatogenic cells (Griswold 1995). During spermatogenesis, germ cells move from the basal compartment to the adluminal compartment (Russell 1977), elongated spermatids are released into the lumen of seminiferous tubules, the cytoplasmic portions of elongated spermatids are shed to form residual bodies before extrusion of differentiated sperm into the lumen (Kerr \& de Kretser 1974, Jegou 1991), and the residual bodies and apoptotic spermatogenic cells are removed by Sertoli cells through phagocytosis (Russell \& Clermont 1977, Chemes 1986). All these events need to be supported by Sertoli cells, and are energy consumable (Kerr 1988, Ueno \& Mori 1990). We found that Sertoli cells produce the high ATP level, which corresponds to the functions of these cells. This result is also in agreement with previous observations that an active energy metabolism occurs within Sertoli cells (Floridi et al. 1983, Ueno \& Mori 1990).

More than $75 \%$ of differentiating spermatogenic cells are estimated to undergo apoptosis under physiological conditions (Oakberg 1956, Huckins 1978, Johnson et al. 1983, Dym 1994). The meaning of the death of such a large proportion of spermatogenic cells is unclear. One explanation is that the number of spermatogenic cells that Sertoli cells can support for maturation is limited, and the elimination of apoptotic cells provides space in the seminiferous epithelium for the development of 
A
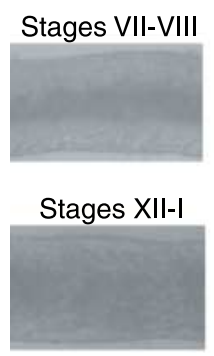

Stages II-VI

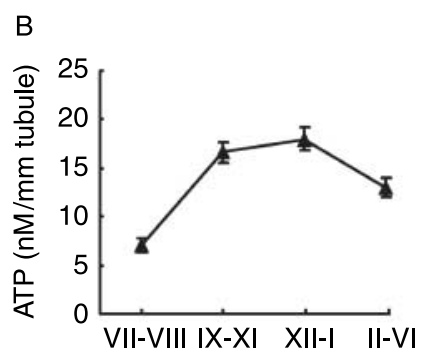

Stages of seminiferous tubules

D

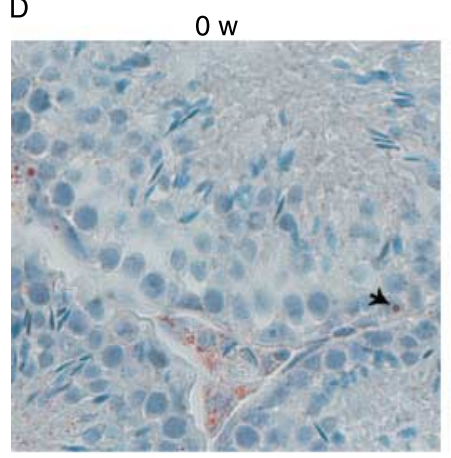

$5 \mathrm{w}$

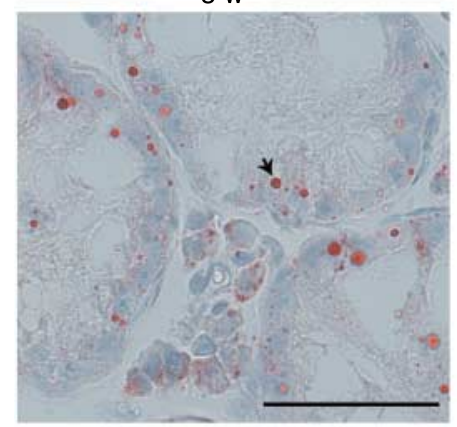

E

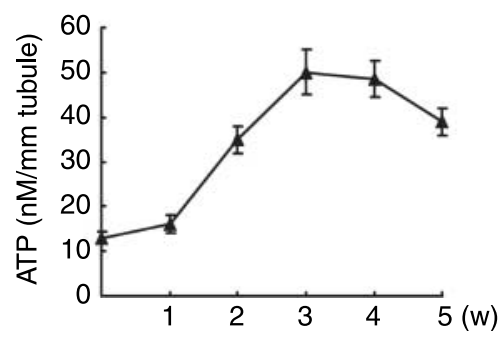

C
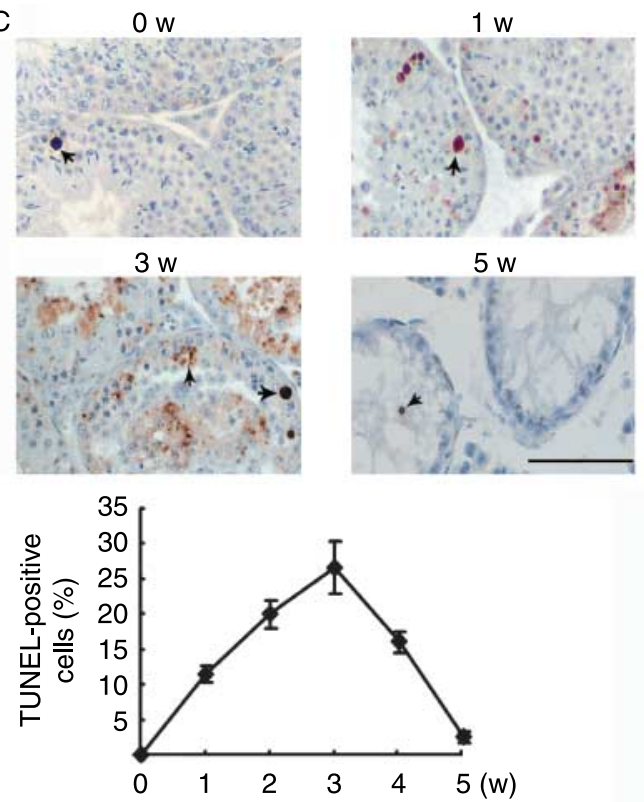

$1 \mathrm{w}$
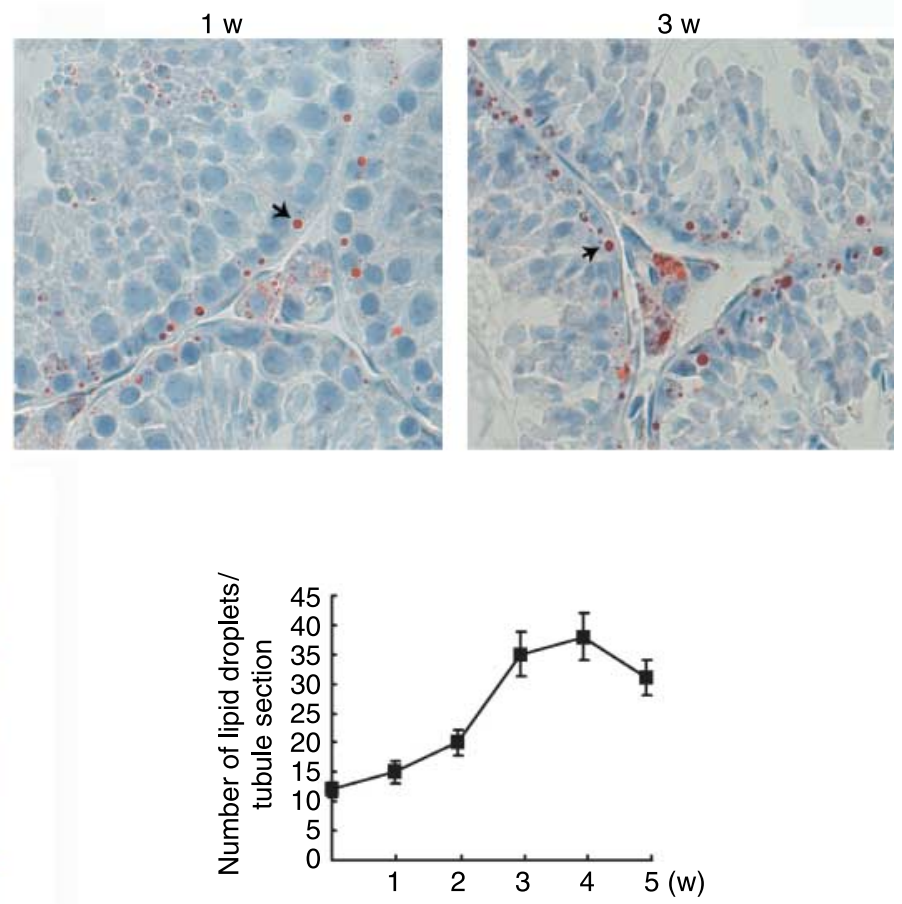
healthy spermatogenic cells. It has been known that phagocytic clearance of apoptotic cells by macrophages is more than mere waste disposal and regulates macrophage function in immune responses (Savill \& Fadok 2000). It is possible that the engulfment of apoptotic spermatogenic cells enables Sertoli cells to support spermatogenesis. How the functions of Sertoli cells can be conferred by the clearance of dying germ cells remains to be clarified. The results in the present study showed that apoptotic spermatogenic cells can be used to produce ATP by Sertoli cells. Notably, the phenomenon that the dying cells are used as an energy source is specific to Sertoli cells, but not to other phagocytes such as macrophages. This energy source could be particularly important for Sertoli cells considering that they are seldom reached by blood circulation in vivo owing to the basement membrane, blood-testis barrier and lacking blood vessels in the seminiferous epithelium. Moreover, this energy resource may confer the meaning of the most spermatogenic cells to undergo apoptosis during spermatogenesis.

ATP is the 'energy currency' of the cells, and plays a central role in many cellular physiological events. Lipids and glucoses are common energy substrates for the production of ATP within cells. Under physiological conditions, most cells predominantly use glucoses to produce ATP, and lipids function as the storage of energy. However, a minority of cell types, such as adiocytes and myocardial cells, actively utilized lipids, but not glucoses, to produce ATP (Crass 1972, 1977, Saddik \& Lopaschuk 1991, Klaus 2004). In this study, by analyzing gene expression of key enzymes participating in lipid $\beta$-oxidation and glycolysis, we found that the expression of LACD, a key enzyme that catabolize LCFAs in the $\beta$-oxidation pathway is significantly increased in Sertoli cells after engulfment of apoptotic germ cells. Moreover, blockade of lipid $\beta$-oxidation pathway by an inhibitor of LACD inhibits ATP production, and the interruption of the glycolysis pathway does not affect ATP synthesis in Sertoli cells during co-culture with apoptotic spermatogenic cells. These observations suggest that Sertoli cells predominantly use lipids to produce ATP through catabolism of LCFAs. The result is in agreement with a recent report that the lipid droplets in Sertoli cells mainly consist of LCFAs (Huyghe et al. 2006). The LCFAs are major energy substrates of cells (Crass 1972, 1977, Saddik \& Lopaschuk 1991, Klaus 2004). The lipid exchange between Sertoli cells and the periphery are currently not well understood. Sertoli cells are largely separated from the peripheral circulation due to the barriers of basement membrane, tight junction between Sertoli cells and lacking of blood capillaries in seminiferous epithelium. The simplest way for Sertoli cells to have sufficient lipids for supporting spermatogenesis would be to recycle the lipid contents in the residual bodies and apoptotic spermatogenic cells.

Massive apoptosis of germ cells also occurs in ovary. It is presumed that only less than $0.1 \%$ of follicles present at the beginning of puberty eventually proceed through ovulation (Tilly et al. 1997). Death of the majority of germ cells is thus an event common to the gametogenesis in both males and females. The significance of gametocytes dying by apoptosis could be an important issue that is worthwhile to investigate further. The study of this issue may open a novel avenue to understand the meaning of germ cell death.

\section{Materials and Methods \\ Animals}

Mice (C57BL/6J) were purchased from the Laboratorial Animal Center of Beijing University (Beijing, China). The mice mutant for Tyro3 RTKs were kindly provided by Dr Lemke (Salk Institute, La Jolla, CA, USA). The animals were maintained in a temperature- and humidity-controlled room on $12 \mathrm{~h}$ light: $12 \mathrm{~h}$ darkness cycle, and had free access to food and water. All the measures taken for the mice were in accordance with guidelines for the Care and Use of Laboratory Animals approved by the Chinese Council on Animal Care.

\section{Isolation of Sertoli, interstitial, and spermatogenic cells}

Three-week-old mice were used for isolation of testicular cells based on a procedure described previously (Wang et al. 2006). Briefly, decapsulated testes were incubated in D-Hanks' solution with $0.5 \mathrm{mg} / \mathrm{ml}$ collagenase (Sigma) at room temperature for $15 \mathrm{~min}$ with gentle oscillation to isolate interstitial cells. After passing through $100 \mu \mathrm{m}$ copper meshes, the interstitial cells were collected by centrifugation at $800 \mathrm{~g}$ for 10 min and cultured in DMEM and Ham's F12 medium (F12/ DMEM; Gibco) supplemented $10 \% \mathrm{FCS}$ at $32{ }^{\circ} \mathrm{C}$ with $5 \% \mathrm{CO}_{2}$ in air. The interstitial cells contain mainly Leydig cells (about $70 \%$ ), macrophages (about 20\%) and a minority of blood cells and myofibroblasts. The seminiferous tubules were resuspended in the collagenase at room temperature for $20 \mathrm{~min}$ to remove myoid cells. The tubules were then incubated in D-Hanks' solution with $0.5 \mathrm{mg} / \mathrm{ml}$ hyaluronidase (Sigma) for 15 min with oscillation. The dispersed cells were cultured in F12/DMEM containing 10\% FCS in a humidified atmosphere with $5 \% \mathrm{CO}_{2}$ at $32{ }^{\circ} \mathrm{C}$. At $24 \mathrm{~h}$ after culture, the spermatogenic

\footnotetext{
Figure 4 In vivo analysis of ATP production in seminiferous tubules. (A) Representative images of different staged tubules under the stereomicroscope. (B) ATP level of the staged tubules was determined by a luciferin-luciferase assay. The high levels of ATP production were observed in tubules of stages IX-XI and XII-I, where the phagocytosis of residual bodies by Sertoli cells occurs. (C) Apoptosis analysis of spermatogenic cells in busulfan-treated testes by TUNEL assay. TUNEL-positive cells are shown in brown (arrowheads). (D) Analysis of lipid droplet formation in Sertoli cells after busulfan treatment. Lipid droplets stained by ORO are shown in red (arrowheads). (E) Dynamics of ATP production in seminiferous tubules after busulfan treatment. The data are represented as mean \pm s.D. of three experiments. Bar $=100 \mu \mathrm{m}$.
} 

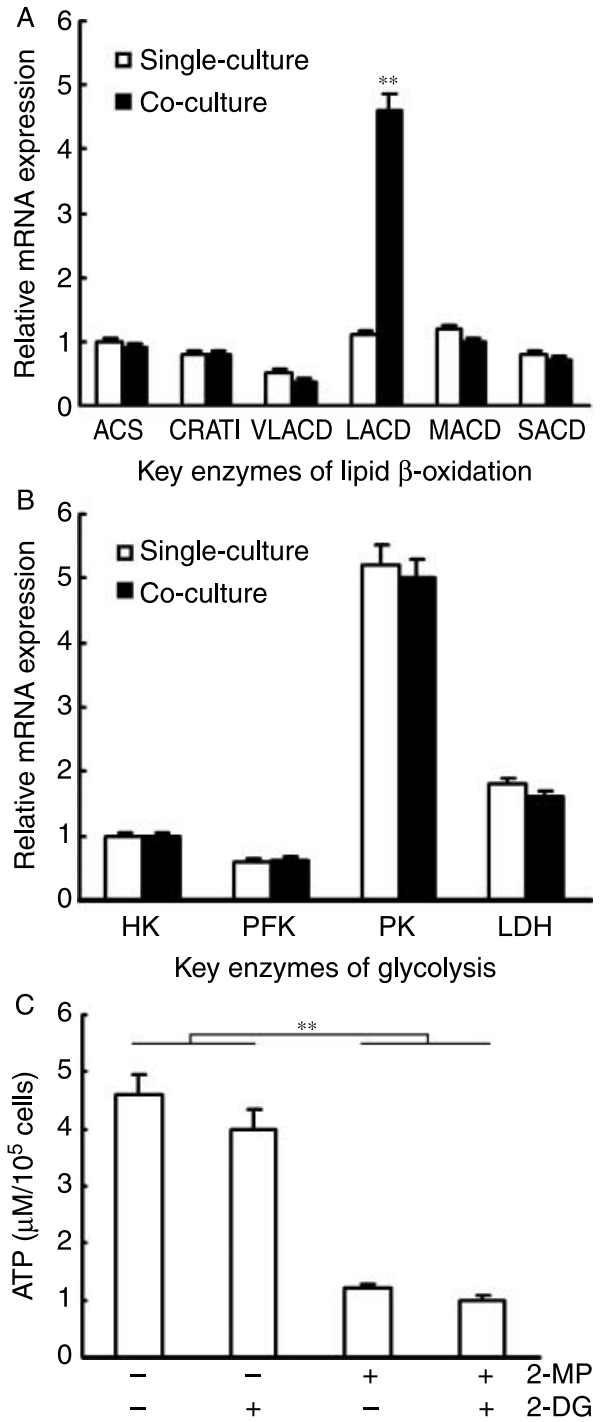

Figure 5 Expression of key enzyme genes involved in metabolism in Sertoli cells. Total RNA from Sertoli cells before and after phagocytosis of apoptotic germ cells was analyzed by real-time RT-PCR. (A) The enzymes involved in lipid $\beta$-oxidation pathway: acyl-CoA synthase (ACS), carnitine acyltransferase I (CRATI), very long-chain acyl-CoA dehydrogenase (VLACD), long-chain acyl-CoA dehydrogenase (LACD), medium-chain acyl-CoA dehydrogenase (MACD) and short-chain acylCoA dehydrogenase (SACD). (B) The enzymes involved in glycolysis: The hexokinase (HK), phosphofructokinase (PFK), pyruvate kinase (PK), and lactate dehydrogenase (LDH). (C) During the co-culture of Sertoli cells and apoptotic spermatogenic cells, the lipid $\beta$-oxidation was blocked by 2-mercaptoacetate (2-MP), and the glycolysis was blocked by 2-deoxyglucose (2-DG). ATP production was significantly inhibited by blocking $\beta$-oxidative pathway. The data are mean \pm s.E.M. of three experiments. ${ }^{* *} P<0.01$.

cells that did not adhered on culture dishes were collected for inducing spontaneous apoptosis by culturing for additional 2 days based on a previous description (Shiratsuchi et al. 1997), and those adhering to Sertoli cells were treated with a hypotonic solution ( $20 \mathrm{mM}$ Tris, $\mathrm{pH} 7.4$ ) for $1 \mathrm{~min}$. Twentyfour hours later, the Sertoli cells were collected and subjected to experiments. The purity of Sertoli cells was more than $95 \%$
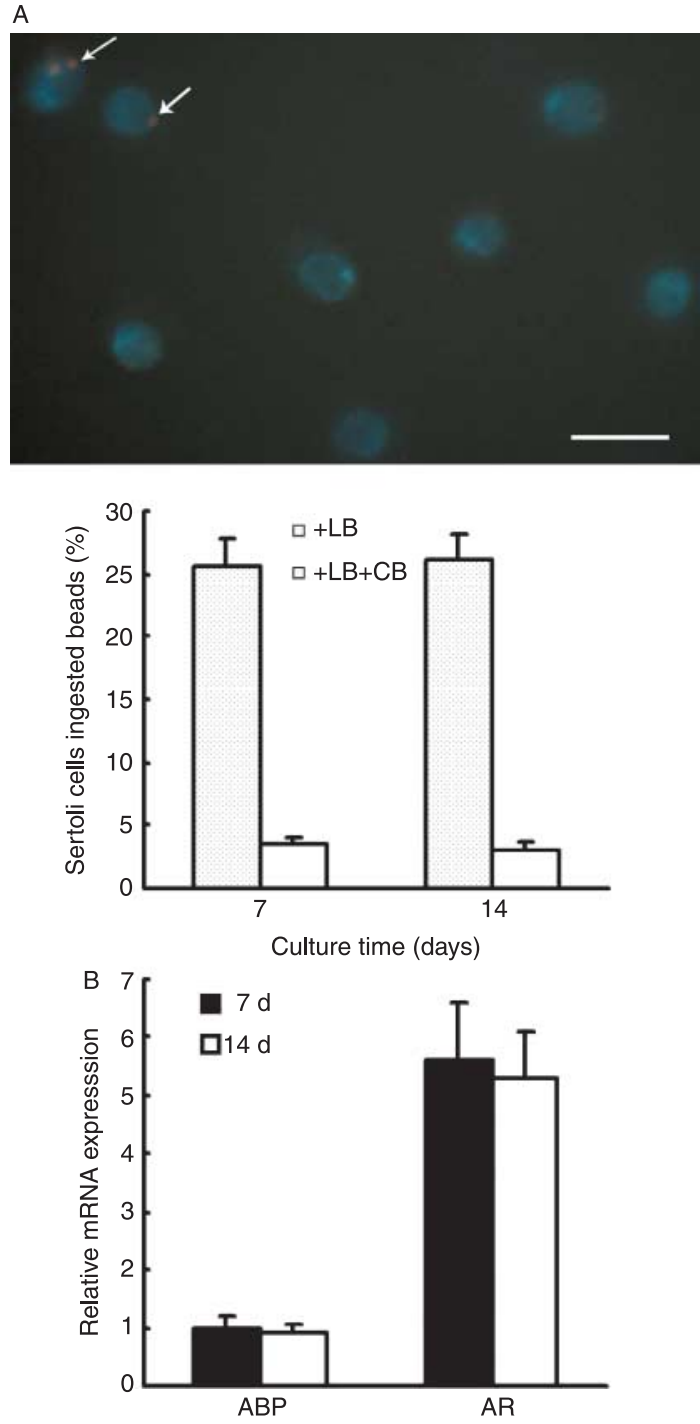

Figure 6 Characterizations of Sertoli cells. (A) Phagocytosis of latex beads (LB) by Sertoli cells. At $6 \mathrm{~h}$ after co-culture, cells were vigorously washed to remove the beads bound on the surface of the cells. Sertoli cells engulfed latex beads (arrows) were identified under a fluorescence microscope (upper panel), and the ratio of the Sertoli cells having internalized latex beads (lower panel). Inhibition of phagocytosis by cytochalasin B (CB) was performed parallely. (B) Gene expression of androgen binding protein (ABP) and androgen receptor (AR) by Sertoli cells during culture. The results were expressed as the mean \pm s.D. of three tests. Bar $=20 \mu \mathrm{m}$.

based on the immunostaining of Wilms' tumor nuclear protein 1 (WT1, a marker of Sertoli cells), and the purity of germ cells was more than $98 \%$ based on morphological analysis.

\section{Isolation of epididymal epithelial cells}

The epididymal epithelial cell culture was prepared as previously described (Moore et al. 1986). Epididymal tissue was obtained from 8-week old mice and washed in PBS to remove blood. The caput epididymidis were minced into small fragments followed by three rinses in PBS to remove 
Table 2 Primers used for real-time RT-PCR.

\begin{tabular}{|c|c|}
\hline Target genes & Primer pair $\left(5^{\prime}-3^{\prime}\right.$, forward and reverse) \\
\hline \multirow[t]{2}{*}{ ACS } & TATGGGCTGACAGAATCATG \\
\hline & CAACTCTTCCAGTAGTGTAG \\
\hline \multirow{2}{*}{ CRATI } & AGCTGGCATACTACAGGATCTATGG \\
\hline & AGGTGAAACATGCGCAGAGA \\
\hline \multirow[t]{2}{*}{ VLACD } & CAGGGATTCAAAGACTTCCAGATAG \\
\hline & GCCGCCTCCGAGCAA \\
\hline \multirow[t]{2}{*}{ LACD } & СТCAATGGAAGCAAGGTGTTCA \\
\hline & GCCACGACGATCACGAGAT \\
\hline \multirow[t]{2}{*}{ MACD } & GCCAATGATGTGTGCTTACTGTGT \\
\hline & ATGGCCGCCACATCAGA \\
\hline \multirow[t]{2}{*}{ SACD } & CGCCACGGTGGTATTTGC \\
\hline & AGGAAGGCACTGATACCCTTGTT \\
\hline \multirow[t]{2}{*}{ HK } & CAGATCGAGAGTGACCGATTAGC \\
\hline & TTAGACCCAGCTGCTGAAGGAT \\
\hline \multirow[t]{2}{*}{ PFK } & СТССССТTСТАGGTTTСССТGTA \\
\hline & GCTCCTGGCCACAGCTGAT \\
\hline \multirow[t]{2}{*}{ PK } & TTGACТСТGСССССАТСАС \\
\hline & GCAGGCCCAATGGTACAAAT \\
\hline \multirow[t]{2}{*}{$\mathrm{LDH}$} & GGACTTGGCGGATGAGCTT \\
\hline & ATCTCGCCCTTGAGTTTGTCTT \\
\hline \multirow[t]{2}{*}{$\mathrm{ABP}$} & AGCCAGCCGGACATGTTG \\
\hline & TGGTGAGGAGGCAATTGGTT \\
\hline \multirow[t]{2}{*}{ AR } & TGGCGGTCCTTCACTAATGTC \\
\hline & TTGAAAACCAAGTCAGGTGCAA \\
\hline \multirow[t]{2}{*}{$28 \mathrm{~S}$ rRNA } & TTGAAAATCCGGGGGAGAG \\
\hline & ACATTGTTCCAACATGCCAG \\
\hline
\end{tabular}

ACS, acyl-CoA synthase; CRATI, carnitine acyltransferase I; VLACD, very long-chain acyl-CoA dehydrogenase; $L A C D$, long-chain acyl-CoA dehydrogenase; MACD, medium-chain acyl-CoA dehydrogenase; SACD, short-chain acyl-CoA dehydrogenase; HK, hexokinase; PFK, phosphofructokinase; $\mathrm{PK}$, pyruvate kinase; $\mathrm{LDH}$, lactate dehydrogenase; $\mathrm{ABP}$, androgen binding protein; $\mathrm{AR}$, androgen receptor; $28 \mathrm{~S}$ rRNA, $28 \mathrm{~S}$ ribosome RNA.

spermatozoa. The dissected epididymal fragments were then incubated in D-Hanks' solution containing $1 \mathrm{mg} / \mathrm{ml}$ collagenase (Sigma) at room temperature for $20 \mathrm{~min}$. The dispersed cells were cultured in minimum essential medium with Earle's salts (Gibco) containing 10\% fetal bovine serum in a humidified atmosphere with $5 \% \mathrm{CO}_{2}$ at $32{ }^{\circ} \mathrm{C}$. Three days latter, the epididymal epithelial cells formed monolayer and subjected to experiments. The purity of epididymal epithelial cells was more than $90 \%$ based on this procedure (Olson et al. 1983).

\section{Isolation of macrophages}

The peritoneal macrophages were isolated based on a previous approach (Chong et al. 2005). Mouse macrophages were collected from peritoneal cavities by lavage with $5 \mathrm{ml}$ cold PBS containing $2 \%$ FCS. The cells were maintained in DMEM containing $10 \%$ FCS at $37^{\circ} \mathrm{C}$ in a humidified atmosphere with $5 \% \mathrm{CO}_{2}$. After $2 \mathrm{~h}$, suspending cells were removed by washing with PBS, and macrophages attached on dishes were subjected to experiments. The purity of the macrophages was more than $95 \%$ estimated by immunostaining of F4/80 (a macrophage marker).

\section{Phagocytosis assay}

The phagocytosis assay was performed based on a previous protocol (Wang et al. 2006). Briefly, Sertoli cells or macrophages were co-cultured with apoptotic germ cells, living germ cells, FITC-labeled inactivated bacteria (E. coli TOP10) and latex beads (Polysciences Inc., Warrington, PA, USA) respectively. It is known that Sertoli cells and macrophages are phagocytic cells, and most interstitial cells and epididymal cells are non-phagocytic cells. At $24 \mathrm{~h}$ after co-culture, the phagocytosis and ATP production by the phagocytes were detected. To distinguish the phagocytic from non-phagocytic Sertoli cells, lipid droplet formation was determined by ORO staining that can be a criterion to evaluate phagocytosis of apoptotic germ cells by phagocytes (Wang et al. 2006). Fluorescent-labeled bacteria and latex beads engulfed by the phagocytes were examined under a fluorescence microscope (IX-71, Olympus).

\section{ATP measurement}

The cellular ATP was measured by a modified luciferinluciferase assay (Yang et al. 2002). Briefly, the cells were collected and transferred to Enppendorf tubes $\left(10^{5}\right.$ cells/tube). After centrifugation at $1000 \mathrm{~g}$ for $10 \mathrm{~min}$, the cell pellets were treated with $1 \mathrm{ml}$ boiling deionized $\mathrm{H}_{2} \mathrm{O}$ to release cellular ATP. After centrifugation at $12000 \mathrm{~g}$ for $5 \mathrm{~min}$ at $4{ }^{\circ} \mathrm{C}$, ATP in the supernatant was measured using luciferin-luciferase reagent (Chrono-Log, Havertown, PA, USA) according to the manufacturer's protocol. The ATP level was presented as $\mu \mathrm{M} / 10^{5}$ cells.

To measure ATP level in seminiferous tubules, the tubules were isolated under a stereomicroscope and cut into fragments of $2 \mathrm{~mm}$ in length according to protocols previously described (Chen et al. 2003). The fragments of the tubules were subjected to ATP measurement. The ATP level was presented as $\mathrm{nM} / \mathrm{mm}$ tubule.

\section{ORO staining}

The lipid droplets were visualized by ORO staining (Wang et al. 2006). Briefly, Sertoli cells were fixed with $10 \%$ formalin for $40 \mathrm{~min}$, and stained with ORO solution (Sigma, ORO saturated solution in isopropanol: water, 3:2) for $15 \mathrm{~min}$. The background staining was removed by washing the cells with $70 \%$ alcohol for $5 \mathrm{~s}$. The lipid droplets in Sertoli cells were analyzed under a microscope (IX71, Olympus). The area ratio of lipid droplets to cell nucleus was used to evaluate phagocytotic ability of Sertoli cells. In total, 200 Sertoli cells from 5 repeat wells were analyzed for each occasion, and the mean values were presented in results.

\section{In vivo analysis}

To analyze the apoptosis of spermatogenic cells, lipid droplet formation and ATP production in vivo, 15 week old mice were injected i.p. with busulfan (Sigma) at single dose $(40 \mu \mathrm{g} / \mathrm{g})$ as reported previously (Maeda et al. 2002). The testes were removed at $0,1,2,3,4,5$ weeks after the injection, and frozen sections of $8 \mu \mathrm{m}$ thickness were sliced with a microtome (Leica CM 1900; Meyer Instruments, Inc., Wetzlar, Germany). The sections were subjected to ORO or TUNEL staining. The lipid droplets and TUNEL-positive spermatogenic cells were examined on 50 sections of seminiferous tubules for each 
testis. The ATP level of the seminiferous tubules was measured. Each analysis was performed on the testes from three mice, and the mean values were presented in results.

\section{Real-time RT-PCR}

Total RNA was extracted using Trizol reagent (Invitrogen) according to the manufacture's instructions. After treatment with DNase I to remove potential contaminating genomic DNA, the RNA $(0.5 \mu \mathrm{g})$ was reverse transcribed into cDNA. Real-time PCR was performed with Power SYBR Green PCR master mix kit (Applied Biosystems, Foster City, CA, USA) using an ABI PRISM 7300 real-time cycler (Applied Biosystems). The transcript levels of target genes were normalized to $28 \mathrm{~S}$ ribosome RNA. The sequences of primer pairs for real-time PCRs are listed in Table 2.

\section{Statistical analysis}

Data were presented as mean \pm s.E.M. (or S.D.) of at least three experiments. Tukey's Test was used to determine the significance for all comparisons in this study. The calculations were performed with SPSS Version 11.0 statistical software.

\section{Declaration of interest}

There is no conflict of interest that could be perceived as prejudicing the impartiality of the research reported.

\section{Funding}

This work was supported by the National Basic Research Program of China (Grant No. 2006CB504001, 2007CB947504) and National Natural Science Foundation of China (Grant No. 30570678).

\section{Acknowledgements}

We sincerely thank Dr Greg Lemke for providing the $\mathrm{TAM}^{-1-}$ mice.

\section{References}

Chemes H 1986 The phagocytic function of Sertoli cells: a morphological, biochemical, and endocrinological study of lysosomes and acid phosphatase localization in the rat testis. Endocrinology 119 1673-1681.

Chen YM, Lee NP, Mruk DD, Lee WM \& Cheng CY 2003 Fer kinase/FerT and adherens junction dynamics in the testis: an in vitro and in vivo study. Biology of Reproduction 69 656-672.

Chong MM, Metcalf D, Jamieson E, Alexander WS \& Kay TW 2005 Suppressor of cytokine signaling- 1 in T cells and macrophages is critical for preventing lethal inflammation. Blood 106 1668-1675.

Chung S, Wang SP, Pan L, Mitchell G, Trasler J \& Hermo L 2001 Infertility and testicular defects in hormone-sensitive lipase-deficient mice. Endocrinology 142 4272-4281.

Crass MF III 1972 Exogenous substrate effects on endogenous lipid metabolism in the working rat heart. Biochimica et Biophysica Acta $28071-81$.

Crass MF III 1977 Regulation of triglyceride metabolism in the isotopically prelabeled perfused heart. Federation Proceedings 36 1995-1999.
Dym M 1994 Spermatogonial stem cells of the testis. PNAS 91 11287-11289.

Floridi A, Marcante ML, D'Atri S, Feriozzi R, Menichini R, Citro G, Cioli V \& De Martino C 1983 Energy metabolism of normal and lonidaminetreated Sertoli cells of rats. Experimental and Molecular Pathology 38 $137-147$.

Griswold MD 1995 Interactions between germ cells and Sertoli cells in the testis. Biology of Reproduction 52 211-216.

Huckins C 1978 The morphology and kinetics of spermatogonial degeneration in normal adult rats: an analysis using a simplified classification of the germinal epithelium. Anatomical Record 190 905-926.

Huyghe S, Schmalbruch H, De Gendt K, Verhoeven G, Guillou F, Van Veldhoven PP \& Baes M 2006 Peroxisomal multifunctional protein 2 is essential for lipid homeostasis in Sertoli cells and male fertility in mice. Endocrinology 147 2228-2236.

Jegou B 1991 Spermatids are regulators of Sertoli cell function. Annals of the New York Academy of Sciences 637 340-353.

Johnson L, Petty CS \& Neaves WB 1983 Further quantification of human spermatogenesis: germ cell loss during postprophase of meiosis and its relationship to daily sperm production. Biology of Reproduction 29 207-215.

Kawasaki Y, Nakagawa A, Nagaosa K, Shiratsuchi A \& Nakanishi Y 2002 Phosphatidylserine binding of class B scavenger receptor type I, a phagocytosis receptor of testicular sertoli cells. Journal of Biological Chemistry 277 27559-27566.

Kerr JB 1988 An ultrastructural and morphometric analysis of the Sertoli cell during the spermatogenic cycle of the rat. Anatomica Embryologica 179 191-203.

Kerr JB \& de Kretser DM 1974 Proceedings: the role of the Sertoli cell in phagocytosis of the residual bodies of spermatids. Journal of Reproduction and Fertility 36 439-440.

Kerr JB \& De Kretser DM 1975 Cyclic variations in Sertoli cell lipid content throughout the spermatogenic cycle in the rat. Journal of Reproduction and Fertility 43 1-8.

Kerr JB, Mayberry RA \& Irby DC 1984 Morphometric studies on lipid inclusions in Sertoli cells during the spermatogenic cycle in the rat. Cell and Tissue Research 236 699-709.

Klaus S 2004 Adipose tissue as a regulator of energy balance. Current Drug Targets 5 241-250.

Maeda Y, Shiratsuchi A, Namiki M \& Nakanishi Y 2002 Inhibition of sperm production in mice by annexin $\mathrm{V}$ microinjected into seminiferous tubules: possible etiology of phagocytic clearance of apoptotic spermatogenic cells and male infertility. Cell Death and Differentiation 9 742-749.

Miething A 1992 Germ-cell death during prespermatogenesis in the testis of the golden hamster. Cell and Tissue Research 267 583-590.

Moore HD, Hartman TD \& Smith CA 1986 In vitro culture of hamster epididymal epithelium and induction of sperm motility. Journal of Reproduction and Fertility 78 327-336.

Nakagawa A, Shiratsuchi A, Tsuda K \& Nakanishi Y 2005 In vivo analysis of phagocytosis of apoptotic cells by testicular Sertoli cells. Molecular Reproduction and Development 71 166-177.

Nakanishi Y \& Shiratsuchi A 2004 Phagocytic removal of apoptotic spermatogenic cells by Sertoli cells: mechanisms and consequences. Biological \& Pharmaceutical Bulletin 27 13-16.

Oakberg EF 1956 A description of spermiogenesis in the mouse and its use in analysis of the cycle of the seminiferous epithelium and germ cell renewal. American Journal of Anatomy 99 391-413.

Olson GE, Jonas-Davies J, Hoffman LH \& Orgebin-Crist MC 1983 Structural features of cultured epithelial cells from the adult rat epididymis. Journal of Andrology 4 347-360.

Pineau C, Le Magueresse B, Courtens JL \& Jegou B 1991 Study in vitro of the phagocytic function of Sertoli cells in the rat. Cell and Tissue Research 264 589-598.

Russell L 1977 Movement of spermatocytes from the basal to the adluminal compartment of the rat testis. American Journal of Anatomy $\mathbf{1 4 8}$ 313-328.

Russell LD \& Clermont Y 1977 Degeneration of germ cells in normal, hypophysectomized and hormone treated hypophysectomized rats. Anatomical Record 187 347-366.

Saddik M \& Lopaschuk GD 1991 Myocardial triglyceride turnover and contribution to energy substrate utilization in isolated working rat hearts. Journal of Biological Chemistry 266 8162-8170. 
Sasso-Cerri E, Giovanoni M, Hayashi H \& Miraglia SM 2001 Morphological alterations and intratubular lipid inclusions as indicative of spermatogenic damage in cimetidine-treated rats. Archives of Andrology 46 5-13.

Savill J \& Fadok V 2000 Corpse clearance defines the meaning of cell death. Nature 407 784-788.

Shiratsuchi A, Umeda M, Ohba Y \& Nakanishi Y 1997 Recognition of phosphatidylserine on the surface of apoptotic spermatogenic cells and subsequent phagocytosis by Sertoli cells of the rat. Journal of Biological Chemistry 272 2354-2358.

Shiratsuchi A, Kawasaki Y, Ikemoto M, Arai H \& Nakanishi Y 1999 Role of class B scavenger receptor type I in phagocytosis of apoptotic rat spermatogenic cells by Sertoli cells. Journal of Biological Chemistry 274 5901-5908.

Tilly JL, Tilly KI \& Perez GI 1997 The genes of cell death and cellular susceptibility to apoptosis in the ovary: a hypothesis. Cell Death and Differentiation 4 180-187.

Ueno H \& Mori H 1990 Morphometrical analysis of Sertoli cell ultrastructure during the seminiferous epithelial cycle in rats. Biology of Reproduction 43 769-776.
Wang H, Wang H, Xiong W, Chen Y, Ma Q, Ma J, Ge Y \& Han D 2006 Evaluation on the phagocytosis of apoptotic spermatogenic cells by Sertoli cells in vitro through detecting lipid droplet formation by Oil Red O staining. Reproduction 132 485-492.

Xiong W, Chen Y, Wang H, Wang H, Wu H, Lu Q \& Han D 2008 Gas6 and the Tyro 3 receptor tyrosine kinase subfamily regulate the phagocytic function of Sertoli cells. Reproduction 135 77-87.

Yang NC, Ho WM, Chen YH \& Hu ML 2002 A convenient one-step extraction of cellular ATP using boiling water for the luciferin-luciferase assay of ATP. Analytical Biochemistry 306 323-327.

Received 11 August 2008

First decision 10 September 2008

Revised manuscript received 17 October 2008

Accepted 11 December 2008 